\title{
Troppi movimenti intorno al Pendolo di Eco!
}

\author{
Rocco Capozzi
}

Proprio quando sembrava che Il nome della rosa sarebbe uscito, dopo otto anni, dalle best seller lists (tipo hit parade di Tuttolibri), e cioc̀, quando sembrava che il "caso Eco" non avrebbe dominato l'intero arco degli anni '80, ecco apparire Il pendolo di Foucuult (Bompiani, 1988)_un romanzo che rimarrà senz'altro al centro di svariate polemiche per almeno un paio di anni. Quindi, non sbaglia chi voglia affermare che "il romanzo italiano degli anni '80 si chiama Eco."

Chi non è ancora convinto del successo-ovviamente non parlo di quello economico-de La rosa deve solo leggere gli articoli raccolti in riviste e volumi quali SubStance 47 (1985), Saggi su "Il nome della rosa" (Bompiani, 1985) c Naming the Rose, a cura di M. T. Inge (Univ. Press of Mississippi, 1988); oppure l'ottimo saggio critico di Theresa Coletti: Naming the Rose: Eco, Medieval Signs and Modern Theory (Cornell Univ. Press, 1988). Dai titoli di questi articoli c saggi si direbbe che la recezione di Eco sia stata molto piú positiva all'estero che in Italia. Questo, credo, si spiega facilmente se si pensa che in Italia a scagliarsi contro il "caso Eco" siano stati soprattutto i suoi colleghi, e cioè molti critici accademici. Gli scrittori, per la piú parte, o hanno taciuto oppure hanno semplicemente lamentato che Eco ha saputo sfruttare, a casa come all'estero, un'operazione di marketing. Quindi non ci sorprende se Geno Pampaloni non ha incluso La rosa nel suo aggiornamento alla "Storia della letteratura italiana" ne Il Novecento della Garzanti, ritenendo che il romanzo di Eco "è un unicum, il prodotto di una raffinatezza culturale che non può essere presa a modello ... è una pietra tombale su tutta la letteratura precedente" (Epoca, 4 sett. '88, 35). E neppure sorprende che Raboni lo escluda dai suoi "cento romanzi italiani del novecento da Salvare" sostenendo che La rosa sarebbe "un imitazione eccellente della letteratura. Sembra un grande romanzo ma non lo è" (Epoca. p. 32). 
Senza entrare in polemica con un certo settore della critica accademica italiana la quale, per quanto molto dotta, spesso cade in vecchi pregiudizi verso gli scrittori contemporanei-specialmente se questi sono viventi, e peggio ancora se sono apprezzati dal cosiddetto grande pubblico-direi che la critica accademica nordamericana sembra essere molto piú aperta alle novità letteraric. Ma non perché "gli americani" (come spesso si sente dire-facendo di ogni erba un fascio, indifferentemente se questi siano canadesi, inglesi, italiani trapiantati in Nordamerica, ecc.) sono sempre pronti ad applaudire tutto ciò che è kitsch e che va di moda, piuttosto perché in Nordamerica si è meno legati a certe tradizioni che ancora esigono una obbedienza a certi modelli letterari e a certe nozioni di "impegno."

Si sa, ci si aspettava da qualche anno che Umberto Eco scrivesse un secondo romanzo e, dopo il "caso Rosa," quasi tutti sfidavano l'autore a ripetere l'incredibile successo del primo romanzo (e questo Eco lo sapeva). Quello che non si sapeva cra che Eco sarebbe riuscito ad eclissare perfino i titoli di autori come Calvino e Moravia e dei vincitori dei premi letterari come Bufalino e la Loy. E dunque trovo divertente ma indovinatissima la vignetta su La Repubblica (21 agosto) in cui Moravia risponde ad Eco: "Cosa vuoi farci? chiunque pubblica un libro nei prossimi tre anni esce in contemporanea col tuo."

Questa rassegna-recensione (piú rassegna che recensione) intende ricostruire, in parte, ciò a cui si è assistito questa estate dal primo annuncio (15-16 luglio) della imminente presentazione del Pendolo, alla Fiera del Libro di Francoforte, fino ad alcune delle prime recensioni apparse nel mese di ottobre. Ovviamente, le polemiche intorno alla valanga di pubblicità che ha preceduto l'uscita del Pendolo, e il modo in cui la stampa ha trattato l'argomento, quasi come un evento di cronaca, meritano molta piú attenzione di quanto non le si possa dare su queste pagine. Ma per quelli un po' lontani dai quotidiani e settimanali italiani (e cioè per quelli che aspettano l'arrivo di queste informazioni nelle loro biblioteche) vale la pena chiarire un po' questo secondo "caso Eco" che a molti è sembrato un "complotto" di Eco, o dell'editore, assieme a degli amici dell'autore, nel garantire che Il pendolo diventasse subito un "caso." Se Eco ha veramente orchestrato questa spettacolare operazione di marketing del suo romanzo si saprà quanto prima, dato che l'autore stesso ci promette 
di parlarne nel futuro (come ha fatto con le P'ostille al nome della rosa?). Per ora diciamo che Eco e la Bompiani non hanno nulla da imparare dagli americani su come si produce e si vende un supercolossal tipo Guerre stellari, ET, Rocky, ecc.; c cioc̀ con annunci, interviste, servizi-foto, magliette e aggeggi pubblicitari, tutto ciò alcuni mesi prima dell'uscita del prodotto. Ben inteso questo non ha nulla a che vedere con la qualità del prodotto: l'importante è far conoscere e vendere a scatola chiusa ciò che si produce. Ma è proprio cosí con Eco? Chi scrive è del parere che per Eco lá qualità del suo libro è essenziale. Eco, oltre ai suoi indiscussi meriti come semiotico, è indubbiamente tra i piú bravi testimoni ed interpreti à la page di ciò che costituisce la "cultura." Come è fuori dubbio che il Nostro dotto massmediologo si ̀̀ già mostrato maestro nel generare, strutturare, e decostruire un romanzo-soprattutto quello piú filosofico e metafisico, e piú precisamente il tipo di romanzo in cui l'autore può giocare piú liberamente a gatto e topo con i suoi lettori: il detective story. Ma non dimentichiamo che se si parla di best-sellers, per Eco bisogna ritornare indictro di alcuni anni e includere testi come Opera aperta. Dunque sostengo che Eco, convinto di avere in mano un prodotto che vale, ci tiene giustamente che questo venga fatto conosecre a vari tipi di lettori [Nozione ribadita dal Nostro in Sugli specchi c altri saggi, 1985: 105]. Tutto sommato, chi dice che uno scrittore non ci terrebbe a far vendere il suo romanzo? Trovo difficile credere che oggi ci siano ancora scrittori felici puramente del loro atto creativo. Con le teorie letterarie recenti sappiamo fin troppo bene che il lettore è già implicito nell'atto della scrittura e che quindi anche se solo indirettamente, l'autore pensa anche al marketing del proprio prodotto. I saggi di Ferretti, Cadioli, Spinazzola, Benussi, ed altri, ci hanno detto qualcosa dei rapporti autore-testo-mercato-pubblico-lettori dagli anni '60 ad cggi.

Ma veniamo ai nostri montoni, come diceva un illustre francese.

\section{In autunno esce $I l$ pendolo di Eco. Ed è subito news}

- 16 luglio. Sul Corriere della sera appaiono pochissime righe che ripetono essenzialmente l'annuncio rilasciato il giorno prima dal Publishers Weekly, e cioè che ad ottobre sarebbe uscito Il pendolo di Foucault di Eco. È da New York (e non da Roma o da Milano) che parte la grande notizia che riesce a rompere la noia estiva ita- 
liana [Ricordiamo che in Italia, durante il periodo estivo, raramente esce un libro di un grande autore. Ai lettori balneari le grandi case editrici propongono i best-seller tradotti, o semmai i romanzi dei cosiddetti "giovani narratori"]. E quindi è bastato solo l'annuncio dell'imminente pubblicazione del secondo romanzo di Eco per mettere in movimento un pendolo che a mio avviso era in movimento da anni.

Lo stesso giorno su Tuttolibri appare un trafiletto-firmato G. C.dal titolo "Per il Pendolo di Eco già in corsa gli stranieri." Ecco quindi i due piccoli petardi che precipitano la voluta valanga di congetture e di sospetti intorno al secondo romanzo di Eco.

\section{- 31 luglio. (“Aspettando Foucault," Panorama, 114-117). Mau-} rizio Bono ri-eca l'evento del 15-16 luglio richiamando l'attenzione sulle "undici righe di stampa piú analizzate e chiosate delle ultime settimane" specificando di quale pendolo si stia parlando e cioè di quello "strumento che dimostra la rotazione della terra inventato nel 1851 dal filosofo Jean Bernard Léon Foucault" [perché c'era già chi pensava all'altro Foucault de Le parole e le cose]. Bono aggiunge che "se la scarna trama del romanzo di Eco odora di misteriosofia e thriller, anche la trama parallela e reale che è appena iniziata attorno al lancio del libro trova i suoi ingredienti principali nel segreto e nel mistero." Ma ciò che oggi (cioè dopo la lettura del Pendolo) risulta estemamente importante nei commenti di Bono sono i riferimenti ad alcune "piste" (tracce intertestuali) da poter seguire nella trama del Pendolo. Bono menziona la relazione di Eco "L'irrazionale ieri c oggi" (ora in Alfabeta 101: 36-38) che fu letta a Francoforte (guarda caso, alla Buchmesse dell'anno precedente). Relazione, dice bene Bono, in cui in sintesi "Eco segnalava preoccupato il dilagare di un nuovo irrazionalismo, che se ha le suc radici nell'ermetismo oggi produce 'manuali di alchimia, astrologia, divinazione, magia nera' e deliri su 'complotti cosmici e cospirazioni maligne'. Che solo l'esercizio critico può smascherare." Ma forse ancora piú interessante è il riferimento ad una altra relazione sul pensicro esoterico tra umanesimo e rinascimento: "Ermete e lo slittamento del senso"-una relazione letta ad un convegno a Siena nel giugno 1987 (si vedano gli Atti, La cifra e l'immagine, a cura di A. Falassi, Sicna, 1988, 173205). Come scopriremo dal Pendolo, ambedue le ipotesi su queste "piste" sono indovinatissime. 


\section{La bomba esplode!}

- 3 agosto ("Eco, detective nelle trame dei Templari," Corriere della sera). Oreste Del Buono riferisee che "una fuga di notizie rompe il silenzio sul Pendolo, il romanzo piú alteso dell'autunno." Dat Del Buono molti italiani vengono a conosenza del fatto che il 30 luglio sul Corriere del Ticino "è uscito un riassunto, sebbene di seconda mano, di un romanzo che non è riassumibile . .." e che quindi "i patti del silenzio, gli accordi di non parlare de $l l$ pendolo sono messi in crisi." Del Buono si sente quindi autorizzato a parlare liberamente del contenuto del romanzo e ci offre dei precisi accenni ai personaggi, al pendolo del Conservatoire, ai Templari, al Piano, c al computer Abulafia. Del Buono è tra i primi a denunciare, su un giornale nazionale, ciò che è accaduto su un giornale di provincia: un caso di indiscrezioni, o meglio, il tradimento di un segreto. Va da sé che in queste due settimane sono ormai già scattati molti sospetti intorno ad un "segreto" che tanti conoscevano (e di cui forse, come d'accordo, bisognava parlarne solo in segreto o con vaghe allusioni fino ad ottobre?).

\section{Fuori l'autore del "Piano"!}

- 21 agosto. ("Delirio per delirio," La Repubblica). Eco si lascia intervistare da Laura Lilli e parla per la prima (?) volta del suo nuovo romanzo assicurandoci che egli, tutt'altro che "regista" di ciò che sta succedendo intorno al Pendolo, ne è in realtà la "vittima" e promette che un giorno scriverà "uno studio su questo fenomeno" [ecco ufficialmente la promessa che al Pendolo non saranno negate le sue "postille"]. Per quanto la tentazione di citare a lungo da questa ricchissima intervista sia forte, mi limito a mettere a fuoco un paio di risposte che riguardano direttamente il romanzo:

... È la storia di tutti i complotti cosmici che la gente veramente si è rappresentata, dal complotto dei Templari ai Protocolli dei Savi di Sion. È la storia di una ossessione del sospetto che pervade infiniti filoni sotterranei della cultura, della politica, delle mistiche degenerate ... È la storia di un cancro spirituale. L'aspetto thriller, l'angoscia, l'inquietudine, non sono un'impalcatura di sostegno, sono la conseguenza di yuesta psicosi storica della "interpretazione sospettosa" della natura, della società, del mondo, dell'ignoto.

Naturalmente i benpensanti possono leggere in questre parole non 
tanto una spiegazione del Pendolo quanto un riferimento a ciò che circonda il "caso Pendolo." E aggiungo che per i lettori curiosi di sapere se anche qui troveranno molte citazioni l'autore aggiunge:

Tutto quello che dicono i mei personaggi è stato detto da qualcuno, e le citazioni sono lì per ancorare il grottesco, l'incredibile, il delirante alla realtà.

Il pendolo di fatto è una piccola biblioteca di citazioni, a cominciare da quelle piú palesi, e in varie lingue, che precedono, come epigrafi, ognuno dei 120 capitoli.

\section{Chi piú ne ha piú ne metta}

- 21 agosto. L'espresso: "L'Eco del tempo" 78-84. Giuseppe Galasso presenta un interessante commento storico sull'Ordine dei Templari dai giorni della sua fondazione nel 1119. Il direttore editoriale della Bompiani, Mario Andreose, in "Voglia di scoop" risponde telegraficamente ad alcune domande sulla storia dei segreti traditi e nega qualsiasi forma di partecipazione/complotto da parte di Eco o della Bompiani.

- 28 agosto. L'Espresso pubblica in anteprima il primo capitolo del romanzo. Purtroppo c'è già chi comincia a giudicare l'intero Pendolo da queste poche pagine che francamente non sono le piú facili del testo; anzi questo capitolo potrebbe perfino scoraggiare dei lettori. Alle pagine di Eco si aggiungono il disegno dell'“albero dei Sefirot" e una scheda, firmata E. G., in cui leggiamo un brevissimo riassunto di questo romanzo "impossibile a riassumere":

Il racconto di Casaubon si snoda a cannocchiale, in un vertiginoso movimento di flashback. Stili di scrittura diversi, colpi di scena, riflessioni filosofiche, storia, misticismo, impegno civile, viaggi mentali, scorribande temporali dal Medioevo all'oggi, dal Brasile all'europa, dai Templari alla Resistenza si intrecciano in un affresco narrativo appassionante.

- 3 settembre. Tuttolibri. Furio Colombo espone le sue lucide impressioni del romanzo che, non ancora pubblicato, è già diventato "l'avvenimento internazionale, nel mondo del libro." Uno dei suggerimenti piú interessanti di Colombo riguarda la "cultura enciclopedica" nel testo, e cioè l'elemento che identificherei assieme all'interdisciplinarietà e all'intertestualità illimitata, come elemento 
basilare nella narrativa, e nei saggi, di Eco.

- 4 settembre ("Troppo Eco," Epoca, 31-34). Ë il turno di Marco Fini a ricostruire alcuni eventi del lancio del best-seller e a parlarci di alcuni aspetti dell" "itinerario mentale e culturale di Éco" [molto divertente la scheda, in cui si illustra l'estro ludico-comico-ironicosatirico-di Eco]. Ormai aumentano sempre di piú le congetture sul libro piú atteso dell'anno, e Fini pone la questione se effettivamente questa storia dei segreti traditi non possa nuocere all'autore: "Chi ha tradito la consegna del silenzio sul Pendolo. . . ? Gli amici furibondi, l'editore scavalcato, i giornali scatenati, il gioco delle anticipazioni rischia di essere controproducente. Riuscirà Umberto Eco a ripetere il successo del Nome della rosa?"

- 2 ottobre. Panorama, forse prevedendo che L'Espresso sta per fare la stessa cosa in occasione dell'uscita del romanzo, stampa un servizio speciale sul Pendolo con scritti di Paolo Rossi, Omar Cálabrese, Maria Corti c Elemirc Zolla; con due pagine estratte dal romanzo; c con tante illustrazioni. In "Il Piano e il Pendolo" (128-133) Rossi si occupa di occultismo e del mondo della magia, in termini filosofici, attirando la nostra attenzione con la domanda: "Da Ermete Trismegisto alla P2. Dai Templari agli oroscopi. Dalla Cabbala all'antisemitismo. Il pendolo di Foucault rilegge 500 anni di pensiero irrazionale. Magia ed esoterismo albergano ancora fra noi?" Omar Calabrese in "Vedi alla voce Graal" (133-139) ci propone un piccolo dizionario delle sette esoteriche e in fondo ad ogni voce segnala due libri: uno "serio" e un altro "delirante," per coloro che vogliano approfondire le letture sull'argomento. Zolla invece, nella sua breve scheda, mostra il suo disappunto affermando: "La costruzione è astuta, il gioco degli argomenti serrato, la conoscenza della materia esoterica piú che sufficiente, ma per un romanzo ci vorrebbe altro"(141). Molto piú entusiasta si dimostra Maria Corti in "Mr. Borges, suppongo?"(139-143) con degli accenni agli aspetti linguistici e intertestuali del romanzo. Trovo giusto il suggerimento che tra le tante tracce nel Pendolo andrebbe cercato Le's Illuminés di Nerval "dove ci sono i Templari, i Rosacroce, il conte di San Germano, Cagliostro, Cazotte, la Cabbala" [Naturalmente, Corti sa che Eco ha lavorato per molto tempo su Silvie). Qui aggiungerei che se c'è un romanzo italiano molto vicino al Pendolo questo è senz'altro /l pia- 
neta azzurro di Luigi Malerba (anche qui troviamo tanti sospetti di complotti e di attività cospiratrici di sette sotterranee come la Massoneria). Purtroppo, una volta nella rete dell'intertestualità illimitata uno incomincia a pensare a tanti altri titoli come Dio e il computer, di Roberto Vacca (Bompiani, 1984), e i sospetti sulle tracce intertestuali non finiscono piú. Siamo comunque d'accordo con il giudizio conclusivo della Corti che "A differenza del Nome della rosa questa seconda opera ci consegna un autore parimenti teso alla soluzione di questioni tematiche e formali, un vero scrittore a tutto tondo; forse il livello stilistico qui è piú alto, c'è uno stile originale, perché piú spiritualmente ampio e profondo è l'impegno dello scrittore."

\section{"Ecofenomeno." Eco performance. Ecomania. Eco ...}

- 9 ottobre. L'Espresso, con tanto di foto di Eco in copertina (alla Man of the year del Time), proclama il dilagarsi dell'"Ecofenomeno." All'interno troviamo "Il mio piano"-una lunghissima intervista di Ferdinando Adornato arricchita da numerose foto-grazie a cui possiamo seguire Eco, piú da vicino, per le strade di Parigi, nel Conservatoire des arts, e, in gencrale, sui luoghi del romanzo. Poi, come testimonial, seguono i commenti del grande storico Jacques Le Goff, "Il diavolo? È lui, Eco" (108-111). Insomma, tutto come uno spettacolare servizio sulla produzione di un supercolossal, diciamo tipo "Marco Polo," con tante foto sul setting e per le strade di Pekino, interviste a qualche star, e con i commenti di un esperto storico su Marco Polo e/o sulla Cina. Inoltre, per un autore-maestro di mappe, specchi e labirinti, non si poteva non includere "una mappa del piano," o meglio, "una sintetica realizzazione grafica per aiutare i lettori del romanzo di Eco." In realtà, piú che la cartina con i disegnini, ciò che aiuterà molti lettori è senz'altro (specialmente se aggiunto al dizionario di Calabrese, in Panorama) la mini-enciclopedia "A come Abulafia T come Templari" di Roberto Cotroneo su "i protagonisti, i luoghi, le cose."

L'intervista è come una "postilla al Pendolo" che certamente molti critici hanno già sezionato e spulciato per i loro scritti. Menziono peraltro, che anche qui Eco non delude i suoi lettori, parlandoci un po' di tutto: Storia, letteratura, scienza, cinema (e come potevano mancare James Bond e il piano Spectre?), semiologia, Parigi, ecc. Eco precisa che sono ben "millecinquecento" i volumi "alla base del 
Pendolo." E per chi si era perso I'intervista su La Repubblica il Nostro ribadisce, in nuce, lo scopo del suo romanzo:

c’è una malattia che si è impossessata della cultura e della politica della nostra epoca. Per questo motivo bo scritto /l pendole: per denunciarlat. È una "malattia dell'interpretazione" che ha inlluchzato tutto. . . . Il suo nome c̀ Sindrome del Sospetto. Il suo strumento è la Dietrologia: dietro un fatto se ne nasconde un altro piú complesso. . . . La vita viene interpretata come un eterno complotto. Anzi una catena di complotti. . .

\section{Tra le prime recensioni:}

- 15 ottobre. Tuttolibri. Molto positiva la reazione di Lorenzo Mondo in "Nelle oscillazioni di un pendolo Eco cerca la verità": "... un romanzo che colpisce per la padronanza di una ricca $\mathrm{c}$ fantasiosa crudizione, per la sferzante ironia, per la severa melanconia."

- 21 ottobre. Sul Corriere della sera leggiamo un Carlo Bo molto deluso: "Addio, lettori di un tempo che fu." Come sappiamo Carlo Bo è una vera e propria istituzione della terza pagina letteraria, oltre ad essere un maestro nel recensire testi senza necessariamente parlare del contenuto. Qui, forse perché Eco è l'autore di Lector in fabula, Bo preferisce parlare delle difficoltà dei lettori davanti al Pendolo, per poi concludere: "Eco non ha nessuna verità da trasmettere, gli interessa l'idea del messaggio segreto $\mathrm{e}$ non il contenuto del messaggio [... .] Grosso modo potremo dire che il libro di Eco è fatto per un immaginario lettore nuovo... l'autore del Pendolo ha sepolto anche noi lettori del passato, lettori fatti soltanto di memoria del cuore."

- 21 ottobre. Europeo: "Francoforte, la sindrome Foucault. Eco e Narciso" (142-145). Ormai tutti discutono di "Ecomania" c in questo reportage S. Pende e E. Regazzoni raccolgono alcune reazioni di scrittori quali Del Giudice ("perfetta c grottesca messa in scena dei mass media"), Pazzi ("un fatto deliziosamente irrazionale"), Elkam ("Eco ... il Berlusconi della letteratura"), Montefoschi ("Ma poi Eco non è un narratore. È una volpe del computer"), Maraini ("L'effetto Eco non può che spingere all'estero i libri italiani"), Sanguineti ("Eco è il Martin Scorsese della letteratura"). Molto piú pungente c̀ la denuncia di F. M. Ricci contro la recezione di Eco da parte di molti intellettuali italiani: "Invidiosi, morti, schialtati, finiti dall'in- 
vidia. Ecco cosa sono gli intellettuali italiani davanti a Eco. . . . Dicono che il romanzo è troppo difficile, che è inaccessibile. La gente vuole e compra solo cose difficili e inaccessibili."

\section{Una delle "vere" stroncature:}

- 21 ottobre. La Repubblica: "Giocando a dadi con Eco e Foucault." La recensione di Pietro Citati, forse piú che per i suoi commenti (molto ad hominem) su Eco (ben rari quelli sul Pendolo) fa scattare alcune domande tipo: cosa fa Citati ne La Repubblica? Ha lasciato il Corriere? Come mai la recensione comincia in prima pagina (se non sbaglio, ciò si era avverato soltanto con Le lezioni americane di Calvino)? Va notato che il romanzo di Eco e la recensione di Citati, tutt'altro che argomenti di pagina letteraria, vengono presentati in un giornale nazionale come se fossero notizie scottanti di cronaca, da leggersi in prima pagina (questo è già in se stesso un altro "fenomeno" per la critica che si occupa dell'industria della letteratura). È indubbio che Citati diverte con la sua sottile ironia nel chiamare Eco un buffone al livello di "Aristofane, Rabelais e, forse Dostoevskij." Ma dove sono i giudizi seri basati sul testo? Non ̀̀ forse il compito dei recensori quello di scrivere per i lettori, per far conoscere i pregi e i difetti di un'opera? Qui invece sembra che si parli esclusivamente agli addetti ai lavori. Citati conclude che Eco "come tutti i Gurdulù, immagina di essere Joycc. . . . Ma Eco non era Calvino, né Cioran, né tantomeno Borges. Era soltanto Umberto Eco."

Se questo è un esempio della nuova critica militante in Italia, possiamo capire le frustrazioni dei lettori di quotidiani e settimanali che cercano delle indicazioni, una guida, sulla narrativa o sulla poesia italiana degli ultimi venti anni.

I quotidiani e settimanali che, come dicevo, hanno fatto a gara a riprendere e riprodurre in varie forme le notizie di "Ecomania," o dell'"operazione marketing" (o dei "complotti") del Pendolo sono molti, e certamente non mancheranno le segnalazioni bibliografiche di questi scritti in articoli che analizzeranno, con giudizi seri, basati su letture piú approfondite (e piú complete), i pregi e i difetti del secondo romanzo di Eco. Comunque credo che $l l$ pendolo dovrà fare i conti con una critica molto "sospettosa," se non proprio cinica. A prescindere dal fatto che è proprio Eco, con il suo "caso: Il nome 
della rosa, " ad aver intensificato le polemiclie e i preconcetti nei riguardi dei cosiddetti best-sellers (ricordiamo che alla fine di ottobre siamo già alla terza edizione, con piú di 300 mila copie vendute), piú importante, $I l$ pendolo dovrà combattere i pregiudizi snobistici che accompagnano etichette come "romanzo postmoderno fitto di citazioni e con il computer."

In un lavoro in progress sto analizzando alcune delle tante similarità con La rosa: complotti, apocalisse, Chiesa, mondo dei libri c di libri fatti di altri libri, citazioni in varie lingue, intertestualiti, arte di decifrare/decodificare/interpretare segni, interventi dell'autore nella narrazione, digressioni, struttura di un giallo che in realtà è un antigiallo, e cosí via. Spero qui di mostrare perché $l l$ pendolo è anche una riuscitissima operazione ludica e teorico-enciclopedica, proprio del tipo che ci aspetteremmo da una mente, tanto sensibile quanto vorace, come Umberto Eco. Di fatto, sono del parere che tutto ciò ch'egli dice su Huizinga (cfr. "Huizinga e il gioco," in Sugli specchi 283-300) si può applicare molto facilmente agli scritti di "Eco ludens" e alla sua armonia di "gioco, cultura e coscienza morale."

Sappiamo dalle Postille che Eco non ama piú il termine postmoderno, soprattutto perché in Italia, e un po' anche all'estero, viene abusato e spesso malcapito. Ciononostante, considero Il pendolo come un ottimo romanzo postmoderno-enciclopedico e intertestuale-in cui Eco ancora una volta dimostra di non poter resistere alla tentazione di voler dire tutto, richiamando tutto, cercando di parlare a tutti di tutto ciò che ha letto visto e sentito. Anzi, da questo punto di vista direi che $I l$ pendolo è molto piú ricco della Rosa, non solo per le numerosissime "passeggiate inferenziali" che i lettori potranno (dovranno) intraprendere nel seguire Casaubon \& Company e il loro "Piano," ma anche per l'impegno dell'autore nei confronti di una società che da secoli cerca il punto fermo, cerca la verità, solo per perdersi facilmente e continuamente nell' "irrazionale," o peggio ancora, come conferma Lia, in una "lista della lavandaia."

Il pendolo, anche se un po' piú difficile del Nome della rosa, dovrebbe divertire vari livelli di lettori: quelli "modelli" e "competenti" come quelli comuni. E come ne La rosa anche qui ce n'è per tutti: richiami di letteratura o di paraletteratura, di fumetti, di Western, e di tanti film che vanno da The Thing, a Saturday Night fever, a The Raiders of the Lost Ark. 
le allusioni ai film di Bogart (come Falco Maltese e Casablanca), e con la loro parodia di questi da parte di Woody Allen in Play it Again Sam; dopo tutto, Casaubon, eccellente Alter-Eco, ammette ripetutamente di voler essere "il Sam Spade" o "il Marlowe della cultura" $(182,243)$. Certamente non rimarranno delusi i lettori che apprezzano l'arguzia e l'arte di Eco nel trattare abduzioni, congetture, segni e segni di altri segni, criptografia, e letture del mondo come una lettura di un testo. Sembrerebbe pure che Eco si sia divertito, con ironia e parodia, alle spalle dei critici che negli ultimi otto anni non hanno fatto altro che parlare della Rosa come un romanzo fatto con-o, per alcuni, fatto da-un computer (Eco ci assicura che alla fine degli anni '70 non usava ancora i suoi word processors). Ebbene, per questi critici, ecco il computer Abulafia, al centro del Pendolo, strumentale per il romanzo nel romanzo di Belbo, con il suo programma basic, con i suoi files, e perfino con un esempio di stampante DMP (dot matrix printer) 9 puntini (34-35, 37-38) [i files di Belbo, forse per facilitare la lettura, sono invece in letter quality]. Immagino che Eco avrà voluto dire, basta con queste storie del computer, ora vi faccio vedere esattamente come si utilizza il computer per e dentro un romanzo. Ma, tutto sommato, che differenza c'è se Eco ha scritto La rosa con una Olivetti Lettera 44 oppure con un Olivetti M24? Con una stilografica o una penna a sfera?

Eco con Casaubon e Belbo (ma qui essendo "nel piano dei tre" dovremmo includere anche Diotallevi), sa anche fare dell'autoironia della sua passione di voler essere un "Marlowe della cultura," c cioè di un detective che forse ama piú l'arte di fare il detective nel cercare delle verità (grazie a congetture, ipotesi, induzioni, deduzioni, piste che portano su altre piste ecc.) che non le verità in se stesse. E per quanto possa scmbrare banale, oppure un cliché (forse perché Eco ha estratto anche questa frase da qualche testo o film-e quindi ribadendo che è veramente difficile, se non impossiblile, parlare "in modo innocente" - si vedano le Postille) penso che il lettore troverà bellissima la conclusione di Casaubon/Eco nell'affermare che nella vita: "Si capisce tutto quando non c'è piú nulla da capire."

Sempre sul filo della parodia e dell'autorironia, Eco ha inserito al centro del romanzo una discussione sul feuilleton [si vedano innanzitutto le pagine 389-390 dove leggiamo frasi quali: "Io col feuilleton ci giocavo, per passeggiare un poco fuori della vita"; e "Ancora una 
volta, per sfuggire all'inquietudine della Storia, Belbo aveva scritto c rivisitato la vita per interposta scrittura"). E indubbio che queste parole non facciano pensare ad un'operazione postmoderna/citazionale e/o a un romanzo puro divertissement. Cioc̀ dubito che Eco non abbia previsto che tra le tante passeggiate inferenziali da lui piantate, frasi come queste non faranno dire ad alcuni critici: ecco esattamente quello che sta facendo Eco con questo romanzo. Questo perché, come vediamo dalle interviste, Eco sa parlare apertamente non solo dei complotti interni al Pendolo ma anche delle voci dei complotti intorno al Pendolo. E quindi non ci sorprende se nel Pendolo troviamo accenni alle polemiche della "fabbrica del romanzo" e dell" "industria dei best-seller." Eco sa che tutti sánno che il libro oggi è poco piú di un prodotto in mano alle grandi corporations, o alle "joint ventures" (196), e cioè che oggi piú che mai il libro c̀ legato a Wall Street. Questo lo afferma esplicitamente Garamond (il quale, forse per i tanti matrimoni e divorzi tra le grandi case editrici italiane, mi fa pensare a Gar(zanti)-Mond(adori), Einaudi, Rizzoli e Bompiani tutte assieme): "Ma anche l'editoria è un industria, la piú nobile tra le industrie, ma una industria" (195).

Se il libro è oggi il prodotto di un sistema editoriale industrializzato, e se un romanzo deve competere sul/nel/al mercato, come tutti gli altri prodotti, che male c'è se per un buon romanzo come $I l$ pendolo (un riuscitissimo giallo metafisico-enciclopedico sui [Eco] generis) si utilizzano le tecniche piú aggiornate di marketing per farlo conoscere? Una volta aperta la scatola: ai lettori l'ardua sentenza.

University of Toronto 